\title{
First order solutions for the buckling loads of weakened Timoshenko columns
}

\author{
G. Vadillo, J.A. Loya*, J. Fernández-Sáez \\ Department of Continuum Mechanics and Structural Analysis, University Carlos III of Madrid, Avda. de la Universidad, 30. 28911 Leganés, Madrid, Spain
}

Keywords:

Timoshenko cracked columns

Buckling loads

Perturbation method

\begin{abstract}
A B S T R A C T
In this work, closed-form expressions for the buckling loads of weakened Timoshenko columns with different boundary conditions and shear force approaches (proportional to the bending rotation or to the total slope) are presented. The crack model used promotes discontinuities in both transversal displacements and rotation due to bending. To solve the buckling problem, the perturbation method is used, considering that the solutions for both the cracked and the uncracked columns are slightly different. This procedure leads to firstorder closed-form expressions for the buckling loads of the Timoshenko cracked column, which were compared with those found by directly solving the corresponding eigenvalue problem, establishing validity limits for these solutions.
\end{abstract}

\section{Introduction}

The analysis of the stability of columns is a topic of great interest in civil, mechanical, aeronautical, nuclear, and offshore engineering. Buckling is one of the most common modes of instability in column-like structures. Buckling of Euler-Bernoulli columns under various end conditions was discussed by Timoshenko and Gere [1]. In addition, the effect of shear deformation in critical buckling loads, as well as the generalized boundary conditions, has recently been discussed by Aristizabal-Ochoa [2,3].

It is well known that the presence of cracks, which can appear in structures as a consequence of the manufacturing process as well as during service loads, diminishes the stiffness of the structure, leading to greater displacements for those loads as well as decreased buckling loads and natural frequencies.

A widely used method to analyse the mechanical behaviour of damaged (weakened) Euler-Bernoulli columns is to consider them as two columns connected at the cracked section by a rotational spring, whose stiffness is related to the crack size and the geometry of the cross section [4]. This model requires the continuity of displacements, bending moment, and shear force and it promotes a discontinuity in the slope of the column deflection proportional to the bending moment transmitted.

For the case of Timoshenko columns, where the effects of shear deformation and rotary inertia are non-negligible, a discontinuity in the transverse deflection at the cracked section must also be considered. This discontinuity must be taken into account from the analysis of local flexibility of a cracked column element (according to Okamura et al. [5] and Tharp [6]).

For the case of weakened Euler-Bernoulli columns, Wang et al. [7] have determined the exact buckling-load values of weakened columns with several end conditions, using the concept of rotationally restrained junction. Caddemi and Calió [8] have solved the problem of buckling of the multi-cracked Euler-Bernoulli column. The presence of a concentrated crack

\footnotetext{
* Corresponding author. Tel.: +34 916248880; fax: +34 916248331.

E-mail address: jloya@ing.uc3m.es (J.A. Loya).
} 
is modelled considering singularities in the flexural stiffness by means of Dirac's delta distributions. Biondi and Caddemi $[9,10]$ showed that this method is equivalent to the internal rotational spring. Loya et al. [11] proposed first-order closedform expressions derived for the buckling loads from the perturbation method.

For the case of uniform Timoshenko cracked columns, the exact buckling-load values can be determined using the procedure presented by Arboleda-Monsalve et al. [12], Zapata-Medina et al. [13] for single crack and by Li [14] for multistep cracked columns with shear deformation. It should be noted that the discontinuity in the transverse displacement is not taken into account in the aforementioned works. The stability and vibration of a non-uniform Timoshenko column with a single crack has been studied by Takahashi [15], while Li [16] obtained exact solutions for buckling of multi-step non-uniform columns with an arbitrary number of cracks.

In this work, first-order closed-form expressions for the buckling loads of a weakened Timoshenko column with different boundary conditions are presented. The approximate expressions were formulated using the perturbation method, considering that the solutions for the cracked and the uncracked columns are slightly different. This procedure has been used previously to calculate the critical buckling loads of Euler-Bernoulli columns [11] as well as the natural frequencies of bending vibrations of Timoshenko columns [17]. The first-order solutions reached using this method are compared with those found by applying other procedures, establishing validity limits between them.

\section{Problem formulation}

Let us consider an uncracked Timoshenko column of length $L$, uniform cross-section $A$, and moment of inertia about the neutral axis, $I$, subjected to a constant compressive axial load $P$ with specified boundary conditions.

Considering the following relationships between bending moment, $M(x)$, shear force, $Q(x)$, axial load, $P$, transverse deflection $y(x)$, and bending rotation $\varphi(x)$, we find that the transverse and bending equations of equilibrium of the Timoshenko column differential element are

$$
\begin{aligned}
& \frac{\partial Q(x)}{\partial x}=0 \\
& \frac{\partial M(x)}{\partial x}+Q(x)+P \frac{\partial y(x)}{\partial x}=0
\end{aligned}
$$

with

$$
M(x)=E I \frac{\partial \varphi(x)}{\partial x}
$$

and shear force, taken into account in this work according to two different approaches: proportional to the bending rotation $\varphi$ or to the total slope of the member axis $\left(\frac{\partial y(x)}{\partial x}\right)$, as proposed by Timoshenko and Gere [1].

\subsection{Shear component proportional to the bending rotation $\varphi$}

Assuming that

$$
Q(x)=\kappa A G\left(\frac{\partial y(x)}{\partial x}-\varphi(x)\right)-P \varphi(x)
$$

the coupled equilibrium equations are in terms of displacements:

$$
\begin{aligned}
& \kappa A G\left(\frac{\partial^{2} y(x)}{\partial x^{2}}-\frac{\partial \varphi(x)}{\partial x}\right)-P \frac{\partial \varphi(x)}{\partial x}=0 \\
& E I \frac{\partial^{2} \varphi(x)}{\partial x^{2}}+\kappa A G\left(\frac{\partial y(x)}{\partial x}-\varphi(x)\right)-P \varphi(x)+P \frac{\partial y(x)}{\partial x}=0
\end{aligned}
$$

where $E$ is the Young modulus, $G$ the shear modulus and $\kappa$ is a coefficient introduced to account for the geometry-dependent distribution of the shear stress.

Using the new dimensionless variables given by

$$
\xi=\frac{x}{L} \quad Y=\frac{y}{L} \quad F^{2}=\frac{P L^{2}}{E I} \quad s^{2}=\frac{E I}{\kappa A G L^{2}}
$$

we can write Eqs. (5) and (6) as:

$$
\begin{aligned}
& \left(1+F^{2} s^{2}\right) \varphi^{\prime}(\xi)-Y^{\prime \prime}(\xi)=0 \\
& \left(1+F^{2} s^{2}\right)\left(Y^{\prime}(\xi)-\varphi(\xi)\right)+s^{2} \varphi^{\prime \prime}(\xi)=0
\end{aligned}
$$

where $(\cdot)^{\prime}$ represents the derivative with respect to $\xi$. 


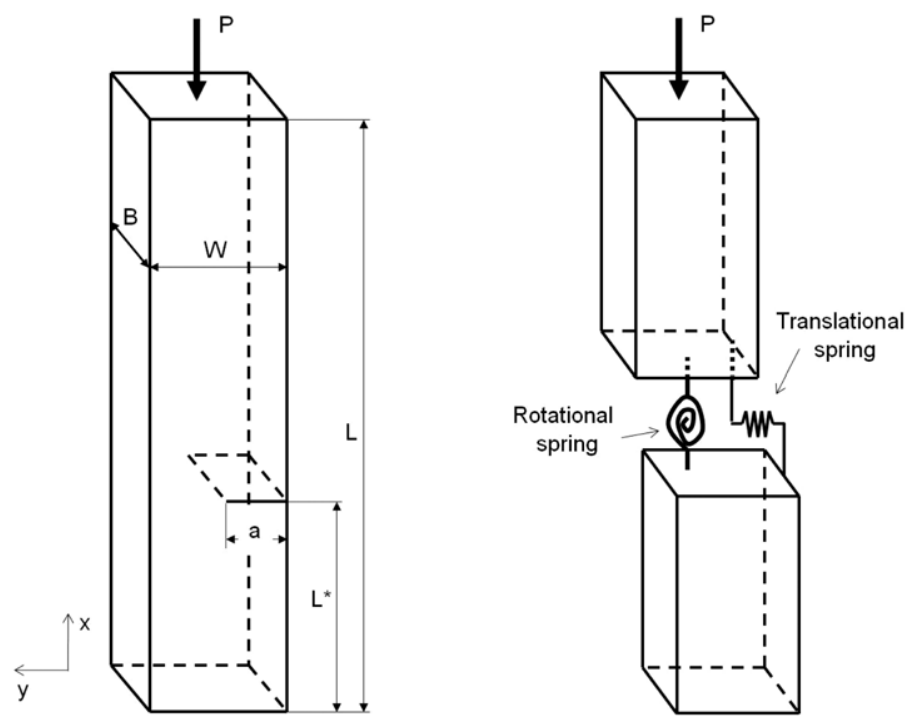

Fig. 1. Model of the cracked column.

Deriving Eq. (7) two times and Eq. (8) once, we get:

$$
\begin{aligned}
& \left(1+F^{2} s^{2}\right) \varphi^{\prime \prime \prime}(\xi)-Y^{I V}(\xi)=0 \\
& \left(1+F^{2} s^{2}\right)\left(Y^{\prime \prime}(\xi)-\varphi^{\prime}(\xi)\right)+s^{2} \varphi^{\prime \prime \prime}(\xi)=0 .
\end{aligned}
$$

Substituting $\varphi^{\prime \prime \prime}(\xi)$ from Eq. (10) into Eq. (9):

$$
\frac{\left(1+F^{2} s^{2}\right)^{2}}{s^{2}}\left(\varphi^{\prime}(\xi)-Y^{\prime \prime}(\xi)\right)-Y^{I V}(\xi)=0
$$

and using Eq. (7), we get the following expression:

$$
Y^{I V}(\xi)+\Omega^{2} Y^{\prime \prime}=0
$$

with

$$
\Omega^{2}=F^{2}\left(1+F^{2} s^{2}\right) .
$$

Then, the general solution of the transverse deflection of the column $Y(\xi)$ takes the form:

$$
Y(\xi)=C_{1} \sin (\Omega \xi)+C_{2} \cos (\Omega \xi)+C_{3} \xi+C_{4} .
$$

Considering the relations between $Y(\xi), \varphi(\xi)$, and its derivatives, given by Eqs. (7) and (8), we can write the rotation due to bending $\varphi(\xi)$ :

$$
\varphi(\xi)=\lambda C_{1} \cos (\Omega \xi)-\lambda C_{2} \sin (\Omega \xi)+C_{3}
$$

where $\lambda=\Omega /\left(1+F^{2} s^{2}\right)$ and $C_{1}, C_{2}, C_{3}, C_{4}$ are constants specified with the boundary conditions at the end supports:

- Fixed end:

$$
Y\left(\xi_{s}\right)=0 ; \quad \varphi\left(\xi_{s}\right)=0 .
$$

- Simply supported end:

$$
Y\left(\xi_{s}\right)=0 ; \quad \varphi^{\prime}\left(\xi_{s}\right)=0 .
$$

- Free end:

$$
\varphi^{\prime}\left(\xi_{s}\right)=0 ; \quad Y^{\prime}\left(\xi_{s}\right)-\left(1+F^{2} s^{2}\right) \varphi\left(\xi_{s}\right)=0
$$

with $\xi_{s}=0$ and $\xi_{s}=1$ at corresponding end supports of the column.

Let us now consider that the column has a crack of depth $a$, always open, and located at a distance $L^{*}$ from the lower support (see Fig. 1).

Following the method proposed by Freund and Hermann [4] and further used by other authors [7,18-25], we can model the cracked column as two columns connected at the cracked section by a rotational spring having stiffness that is related 
to the crack size and the geometry of the cross section. This model leads to discontinuities in transversal displacement and in rotation due to bending being proportional, respectively, to shear force and bending moment transmitted by the cracked section [5,6]:

$$
\begin{aligned}
& \Delta y=\left.\frac{W}{E A} q(\alpha)\left(\kappa A G\left(\frac{d y}{d x}-\varphi\right)-P \varphi\right)\right|_{x=L^{*}} \\
& \Delta \varphi=\left.\frac{W}{E I} \Theta(\alpha) \frac{d \varphi}{d x}\right|_{x=L^{*}}
\end{aligned}
$$

where $\Delta(\cdot)$ represents the jump $(\cdot)_{x=L^{*+}}-(\cdot)_{x=L^{*-}}$ in these equations, $W$ is the width of the column, and $q(\alpha)$ and $\Theta(\alpha)$ are functions that depend on the crack-length to width ratio $\alpha=a / W$ and on the column cross-section geometry. In case of a rectangular cross-section, the function $\Theta(\alpha)$ can be written as [26]

$$
\Theta(\alpha)=2\left(\frac{\alpha}{1-\alpha}\right)^{2}\left(5.93-19.69 \alpha+37.14 \alpha^{2}-35.84 \alpha^{3}+13.12 \alpha^{4}\right)
$$

and the function $q(\alpha)$, obtained from the work by Tharp [6] is given by

$$
q(\alpha)=\left(\frac{\alpha}{1-\alpha}\right)^{2}\left(-0.816+9.80 \alpha-16.492 \alpha^{2}+7.1547 \alpha^{3}+0.3504 \alpha^{4}\right) .
$$

With the use of the new variables:

$$
\beta=\frac{L^{*}}{L} \quad r^{2}=\frac{I}{A L^{2}} ; \quad \eta_{q}=\frac{W}{L} q(\alpha) \quad \eta_{m}=\frac{W}{L} \Theta(\alpha) .
$$

Eqs. (19) and (20) take the form:

$$
\begin{aligned}
& \Delta Y(\xi)=\left.\eta_{q} \frac{r^{2}}{s^{2}}\left(Y^{\prime}(\xi)-\varphi(\xi)-F^{2} s^{2} \varphi(\xi)\right)\right|_{\xi=\beta} \\
& \Delta \varphi(\xi)=\left.\eta_{m} \varphi^{\prime}(\xi)\right|_{\xi=\beta} .
\end{aligned}
$$

Then, the problem consists on solving the following set of equations:

$$
\begin{aligned}
& \left(1+F^{2} s^{2}\right) \varphi_{1}^{\prime}(\xi)-Y_{1}^{\prime \prime}(\xi)=0 \quad 0<\xi<\beta \\
& s^{2} \varphi_{1}^{\prime \prime}(\xi)+\left(1+F^{2} s^{2}\right) Y_{1}^{\prime}(\xi)-\left(1+F^{2} s^{2}\right) \varphi_{1}(\xi)=0 \quad 0<\xi<\beta \\
& \left(1+F^{2} s^{2}\right) \varphi_{2}^{\prime}(\xi)-Y_{2}^{\prime \prime}(\xi)=0 \quad \beta<\xi<1 \\
& s^{2} \varphi_{2}^{\prime \prime}(\xi)+\left(1+F^{2} s^{2}\right) Y_{2}^{\prime}(\xi)-\left(1+F^{2} s^{2}\right) \varphi_{2}(\xi)=0 \quad \beta<\xi<1
\end{aligned}
$$

subjected to the compatibility following conditions at the cracked section $(\xi=\beta)$ :

- Jump in the transverse deflection:

$$
\Delta Y(\beta)=\eta_{q} \frac{r^{2}}{s^{2}}\left[Y^{\prime}(\beta)-\left(1+F^{2} s^{2}\right) \varphi(\beta)\right] .
$$

- Jump in the slope deflection:

$$
\Delta \varphi(\beta)=\eta_{m} \varphi^{\prime}(\beta) .
$$

- Continuity of the bending moment:

$$
\Delta \varphi^{\prime}(\beta)=0 .
$$

- Continuity of the shear force:

$$
\Delta\left[Y^{\prime}(\beta)-\left(1+F^{2} s^{2}\right) \varphi(\beta)\right]=0 .
$$

\subsection{Shear component proportional to the total slope $\frac{\partial y(x)}{\partial x}$}

If the shear-force component is assumed to be proportional to the total slope, then

$$
Q(x)=\kappa A G\left(\frac{\partial y(x)}{\partial x}-\varphi(x)\right)-P \frac{\partial y(x)}{\partial x}
$$


the coupled equations for the equilibrium-state Eqs. (1) and (2) can be written now as

$$
\begin{aligned}
& \kappa A G\left(\frac{\partial^{2} y(x)}{\partial x^{2}}-\frac{\partial \varphi(x)}{\partial x}\right)-P \frac{\partial^{2} y(x)}{\partial x^{2}}=0 \\
& E I \frac{\partial^{2} \varphi(x)}{\partial x^{2}}+\kappa A G\left(\frac{\partial y(x)}{\partial x}-\varphi(x)\right)=0 .
\end{aligned}
$$

If the same procedure as in the first approach is followed, the solution of the problem for the case $F^{2} s^{2}<1$ leads to

$$
Y^{I V}(\xi)+\Omega^{2} Y^{\prime \prime}=0
$$

with

$$
\Omega^{2}=F^{2} /\left(1-F^{2} s^{2}\right)
$$

Then, the general solution of the transverse deflection of the column $Y(\xi)$, takes the form of Eqs. (14)-(15) with $\lambda=\Omega\left(1-F^{2} s^{2}\right)$ and $C_{1}, C_{2}, C_{3}, C_{4}$ obtained from the new boundary conditions:

- Fixed end: $Y\left(\xi_{s}\right)=0 ; \varphi\left(\xi_{s}\right)=0$

- Simply supported end: $Y\left(\xi_{s}\right)=0 ; \varphi^{\prime}\left(\xi_{s}\right)=0$

- Free end: $\varphi^{\prime}\left(\xi_{s}\right)=0 ;\left(1-F^{2} s^{2}\right) Y^{\prime}\left(\xi_{s}\right)-\varphi\left(\xi_{s}\right)=0$

with $\xi_{s}=0$ and $\xi_{s}=1$ at corresponding end supports of the column.

In the case of a cracked column, when the new definition of shear-force of Eq. (34) in the method described before is considered, the problem consists of solving the following set of equations

$$
\begin{array}{ll}
\left(1-F^{2} s^{2}\right) Y_{1}^{\prime \prime}(\xi)-\varphi_{1}^{\prime}(\xi)=0 & 0<\xi<\beta \\
s^{2} \varphi_{1}^{\prime \prime}(\xi)+Y_{1}^{\prime}(\xi)-\varphi_{1}(\xi)=0 & 0<\xi<\beta \\
\left(1-F^{2} s^{2}\right) Y_{2}^{\prime \prime}(\xi)-\varphi_{2}^{\prime}(\xi)=0 & \beta<\xi<1 \\
s^{2} \varphi_{2}^{\prime \prime}(\xi)+Y_{2}^{\prime}(\xi)-\varphi_{2}(\xi)=0 & \beta<\xi<1
\end{array}
$$

subjected to the compatibility conditions at the cracked column:

- Jump in the transverse deflection: $\Delta Y(\beta)=\eta_{q} \frac{r^{2}}{s^{2}}\left[\left(1-F^{2} s^{2}\right) Y^{\prime}(\beta)-\varphi(\beta)\right]$

- Jump in the slope deflection: $\Delta \varphi(\beta)=\eta_{m} \varphi^{\prime}(\beta)$

- Continuity of the bending moment: $\Delta \varphi^{\prime}(\beta)=0$

- Continuity of the shear force: $\Delta\left[\left(1-F^{2} s^{2}\right) Y^{\prime}(\beta)-\varphi(\beta)\right]=0$

and the boundary conditions at each end support.

\section{Direct method of solution}

A direct solution can be achieved by analysing separately the two segments lying on either side of the crack. The solution for each segment $\left(Y_{1}, \varphi_{1}\right)$ on the first part and $\left(Y_{2}, \varphi_{2}\right)$ on the second part of the column can be written as

$$
\begin{aligned}
& Y_{1}(\xi)=C_{1} \sin (\Omega \xi)+C_{2} \cos (\Omega \xi)+C_{3} \xi+C_{4} \quad 0<\xi<\beta \\
& \varphi_{1}(\xi)=\lambda C_{1} \cos (\Omega \xi)-\lambda C_{2} \sin (\Omega \xi)+C_{3} \quad 0<\xi<\beta \\
& Y_{2}(\xi)=C_{5} \sin (\Omega \xi)+C_{6} \cos (\Omega \xi)+C_{7} \xi+C_{8} \quad \beta<\xi<1 \\
& \varphi_{2}(\xi)=\lambda C_{5} \cos (\Omega \xi)-\lambda C_{6} \sin (\Omega \xi)+C_{7} \quad \beta<\xi<1 .
\end{aligned}
$$

The values of the eight constants $C_{1}$ to $C_{8}$ are determined using the four boundary conditions (two for each end) and the four compatibility conditions at the cracked section. The linear algebraic homogeneous system to be solved is

$$
A_{i j} C_{j}=0 \quad(i, j=1, \ldots, 8) .
$$

For non-trivial solutions of $C_{j}$, the determinant of the coefficients' matrix $A_{i j}$ must be zero

$$
\operatorname{det}\left(A_{i j}\right)=0 .
$$

The lower value of $F$ that satisfies the above condition is the first critical buckling load $F_{c}^{*}$. 


\section{First-order perturbative solution}

The perturbation method can be applied to solve Eqs. (26)-(29) and (39)-(42) as alternative to the direct solution. The perturbative solution is reached by extending the method originally proposed by Morassi [19] for the bending vibrations of a cracked Euler-Bernoulli column, under the assumption that the solution for the cracked and uncracked columns are slightly different. Since two springs connecting the two segments of the column have been considered, the solutions can be expanded in a Taylor series with respect to the small parameters, $\varepsilon_{R}$ and $\varepsilon_{T}$, related with the flexibilities of the rotational and transversal springs, respectively. Depending on the shape of the cross-section and on the shape of the crack, the order of smallness of both flexibilities could be different. Thus, for example, the solution for the transversal displacement of the first segment of the column should be written as

$$
Y_{1}(\xi)=Y(\xi)+\varepsilon_{T} V_{1 T}(\xi)+\varepsilon_{R} V_{1 R}(\xi)+O\left(\varepsilon_{T}^{2}, \varepsilon_{R}^{2}\right) .
$$

However, for the special case of the rectangular cross-section, the two flexibilities have the same order on $\alpha(\Theta(\alpha)=$ $\mathrm{O}\left(\alpha^{2}\right)$ and $\left.q(\alpha)=\mathrm{O}\left(\alpha^{2}\right)\right)$ (see Eqs. (21) and (22)) and, therefore, it is reasonable to expand the solution with respect to a single parameter.

Accordingly, the following expansions are introduced:

$$
\begin{array}{ll}
Y_{1}(\xi)=Y(\xi)+\varepsilon V_{1}(\xi)+O\left(\varepsilon^{2}\right) & 0<\xi<\beta \\
\varphi_{1}(\xi)=\varphi(\xi)+\varepsilon \psi_{1}(\xi)+O\left(\varepsilon^{2}\right) & 0<\xi<\beta \\
Y_{2}(\xi)=Y(\xi)+\varepsilon V_{2}(\xi)+O\left(\varepsilon^{2}\right) & \beta<\xi<1 \\
\varphi_{2}(\xi)=\varphi(\xi)+\varepsilon \psi_{2}(\xi)+O\left(\varepsilon^{2}\right) & \beta<\xi<1 \\
F_{c}^{* 2}=F_{c}^{2}+\varepsilon \mu^{2}+O\left(\varepsilon^{2}\right) &
\end{array}
$$

where $\varepsilon$ is a small parameter of the same order as the flexibility of the linear spring representing the crack, $F_{c}$ is the first critical buckling load of the uncracked column, and $V_{1}(\xi), V_{2}(\xi)$, and $\mu^{2}$ are variables of the problem that have to be determined as a part of the solution.

Note that the above expansions are valid for the case of simple eigenvalues. A discussion about the multiplicity of eigenvalues related to the bending vibrations of Timoshenko columns can be found in the works by Geist and McLaughlin [27] and van Rensburg and van der Merwe [28]. In the case of multiple eigenvalues, the standard Taylor expansion must be modified as explained in the classical book by Courant and Hilbert [29].

The mode shapes for displacement, $Y(\xi)$, and slope, $\varphi(\xi)$, for the uncracked Timoshenko column, as well as the first (lower) eigenvalue, $\Omega$, with different boundary conditions have the following expressions:

- Pinned-pinned

$$
\begin{aligned}
& Y(\xi)=C_{1} \sin (\Omega \xi) \\
& \varphi(\xi)=\lambda C_{1} \cos (\Omega \xi) \\
& \Omega=\pi .
\end{aligned}
$$

- Clamped-pinned

$$
\begin{aligned}
& Y(\xi)=-C_{1} \sin (\Omega(1-\xi))+C_{1} \sin (\Omega) \cdot(1-\xi) \\
& \varphi(\xi)=\lambda C_{1} \cos (\Omega(1-\xi))-C_{1} \sin (\Omega) \\
& \Omega=\arctan (\lambda) .
\end{aligned}
$$

- Clamped-free

$$
\begin{aligned}
& Y(\xi)=C_{2} \cos (\Omega \xi)-C_{2} \\
& \varphi(\xi)=-\lambda C_{2} \sin (\Omega \xi) \\
& \Omega=\frac{\pi}{2} .
\end{aligned}
$$

- Clamped-clamped

$$
\begin{aligned}
& Y(\xi)=C_{2} \cos (\Omega \xi)-C_{2} \\
& \varphi(\xi)=-\lambda C_{2} \sin (\Omega \xi) \\
& \Omega=2 \pi
\end{aligned}
$$

where $\Omega$ and $\lambda$ have the expressions corresponding to the two different shear force definitions considered and $C_{1}$ and $C_{2}$ are arbitrary constants. Following a standard procedure (see [28] for instance), it can be seen that the first eigenvalue for all the cases considered are simple eigenvalues and then, the expansions given by Eqs. (50)-(54) are valid. 


\subsection{Shear component proportional to the bending rotation}

If the first-order terms are kept after substituting the new variables into Eqs. (26)-(29):

$$
\begin{array}{ll}
\left(1+F_{c}^{2} s^{2}\right) \psi_{1}^{\prime}(\xi)-V_{1}^{\prime \prime}(\xi)=-\mu^{2} s^{2} \varphi^{\prime}(\xi) \quad 0<\xi<\beta & \\
\left(1+F_{c}^{2} s^{2}\right)\left[V_{1}^{\prime}(\xi)-\psi_{1}(\xi)\right]+s^{2} \psi_{1}^{\prime \prime}(\xi)=-\mu^{2} s^{2}\left[Y^{\prime}(\xi)-\varphi(\xi)\right] & 0<\xi<\beta \\
\left(1+F_{c}^{2} s^{2}\right) \psi_{2}^{\prime}(\xi)-V_{2}^{\prime \prime}(\xi)=-\mu^{2} s^{2} \varphi^{\prime}(\xi) \quad \beta<\xi<1 & \\
\left(1+F_{c}^{2} s^{2}\right)\left[V_{2}^{\prime}(\xi)-\psi_{2}(\xi)\right]+s^{2} \psi_{2}^{\prime \prime}(\xi)=-\mu^{2} s^{2}\left[Y^{\prime}(\xi)-\varphi(\xi)\right] & \beta<\xi<1 .
\end{array}
$$

The above equations are solved, including the following boundary and compatibility conditions:

(a) Boundary conditions:

- Fixed end:

$$
V_{i}\left(\xi_{s}\right)=0, \quad \psi_{i}\left(\xi_{s}\right)=0
$$

- Simply supported end:

- Free end:

$$
V_{i}\left(\xi_{s}\right)=0, \quad \psi_{i}^{\prime}\left(\xi_{s}\right)=0
$$

$$
\psi_{i}^{\prime}\left(\xi_{s}\right)=0, \quad V_{i}^{\prime}\left(\xi_{s}\right)-\left(1+F_{c}^{2} s^{2}\right) \psi_{i}\left(\xi_{s}\right)=\mu^{2} s^{2} \varphi\left(\xi_{s}\right)
$$

with $i=1$ and $\xi_{s}=0$ for the first part of the column, and $i=2$ and $\xi_{s}=1$ for the second one.

(b) Compatibility conditions at the cracked section $(\xi=\beta)$ :

- Jump in the transverse deflection:

$$
V_{2}(\beta)-V_{1}(\beta)=\frac{1}{\varepsilon} \eta_{q} \frac{r^{2}}{s^{2}}\left[Y^{\prime}(\beta)-\left(1+F_{c}^{2} s^{2}\right) \varphi(\beta)\right] .
$$

- Jump in the slope deflection:

$$
\psi_{2}(\beta)-\psi_{1}(\beta)=\frac{1}{\varepsilon} \eta_{m} \varphi^{\prime}(\beta) .
$$

- Continuity of the bending moment:

$$
\psi_{1}^{\prime}(\beta)=\psi_{2}^{\prime}(\beta) \text {. }
$$

- Continuity of the shear force:

$$
V_{1}^{\prime}(\beta)-\left(1+F_{c}^{2} s^{2}\right) \psi_{1}(\beta)=V_{2}^{\prime}(\beta)-\left(1+F_{c}^{2} s^{2}\right) \psi_{2}(\beta) .
$$

By multiplying Eqs. (60) and (62) by $Y(\xi)$, and Eqs. (61) and (63) by $(-\varphi(\xi))$, adding the results found, and integrating over the whole length of the column, we get the following expression:

$$
\begin{aligned}
& \int_{0}^{\beta}\left[\left(1+F_{c}^{2} s^{2}\right) \psi_{1}^{\prime}(\xi)-V_{1}^{\prime \prime}(\xi)\right] Y(\xi)-\int_{0}^{\beta}\left[\left(1+F_{c}^{2} s^{2}\right)\left[V_{1}^{\prime}(\xi)-\psi_{1}(\xi)\right]+s^{2} \psi_{1}^{\prime \prime}(\xi)\right] \varphi(\xi) \\
& \quad+\int_{\beta}^{1}\left[\left(1+F_{c}^{2} s^{2}\right) \psi_{2}^{\prime}(\xi)-V_{2}^{\prime \prime}(\xi)\right] Y(\xi)-\int_{\beta}^{1}\left[\left(1+F_{c}^{2} s^{2}\right)\left[V_{2}^{\prime}(\xi)-\psi_{2}(\xi)\right]+s^{2} \psi_{2}^{\prime \prime}(\xi)\right] \varphi(\xi) \\
& =-\mu^{2} s^{2} \int_{0}^{1}\left[Y(\xi) \varphi^{\prime}(\xi)-\varphi(\xi) Y^{\prime}(\xi)+\varphi^{2}(\xi)\right] d \xi .
\end{aligned}
$$

When we integrate by parts, Eq. (71) becomes:

$$
H_{1}+H_{2}+H_{3}+H_{4}=-\mu^{2} s^{2} H_{5}
$$

being:

$$
\begin{aligned}
H_{1}= & \left(1+F_{c}^{2} s^{2}\right) \int_{0}^{\beta}\left[V_{1}(\xi) \varphi^{\prime}(\xi)+\psi_{1}(\xi) \varphi(\xi)-\psi_{1}(\xi) Y^{\prime}(\xi) d \xi\right] \\
& -\int_{0}^{\beta} V_{1}(\xi) Y^{\prime \prime}(\xi) d \xi-s^{2} \int_{0}^{\beta} \psi_{1}(\xi) \varphi^{\prime \prime}(\xi) d \xi-\int_{\beta}^{1} V_{2}(\xi) Y^{\prime \prime}(\xi) d \xi \\
& -s^{2} \int_{\beta}^{1} \psi_{2}(\xi) \varphi^{\prime \prime}(\xi) d \xi+\left(1+F_{c}^{2} s^{2}\right) \int_{\beta}^{1}\left[V_{2}(\xi) \varphi^{\prime}(\xi)+\psi_{2}(\xi) \varphi(\xi)-\psi_{2}(\xi) Y^{\prime}(\xi)\right] d \xi \\
H_{2}= & -\left(1+F_{c}^{2} s^{2}\right) \psi_{1}(0) Y(0)+V_{1}^{\prime}(0) Y(0)-V_{1}(0) Y^{\prime}(0)+s^{2} \psi_{1}^{\prime}(0) \varphi(0) \\
& -s^{2} \psi_{1}(0) \varphi^{\prime}(0)+\left(1+F_{c}^{2} s^{2}\right) V_{1}(0) \varphi(0) \\
H_{3}= & \left(1+F_{c}^{2} s^{2}\right) \psi_{1}(\beta) Y(\beta)-V_{1}^{\prime}(\beta) Y(\beta)+V_{1}(\beta) Y^{\prime}(\beta)-s^{2} \psi_{1}^{\prime}(\beta) \varphi(\beta)+s^{2} \psi_{1}(\beta) \varphi^{\prime}(\beta) \\
& -\left(1+F_{c}^{2} s^{2}\right) V_{1}(\beta) \varphi(\beta)-\left(1+F_{c}^{2} s^{2}\right) \psi_{2}(\beta) Y(\beta)+V_{2}^{\prime}(\beta) Y(\beta)-V_{2}(\beta) Y^{\prime}(\beta)+s^{2} \psi_{2}^{\prime}(\beta) \varphi(\beta) \\
& -s^{2} \psi_{2}(\beta) \varphi^{\prime}(\beta)+\left(1+F_{c}^{2} s^{2}\right) V_{2}(\beta) \varphi(\beta) \\
&
\end{aligned}
$$




$$
\begin{aligned}
& H_{4}=\left(1+F_{c}^{2} s^{2}\right) \psi_{2}(1) Y(1)-V_{2}^{\prime}(1) Y(1)+V_{2}(1) Y^{\prime}(1)-s^{2} \psi_{2}^{\prime}(1) \varphi(1)+s^{2} \psi_{2}(1) \varphi^{\prime}(1)-\left(1+F_{c}^{2} s^{2}\right) V_{2}(1) \varphi(1) \\
& H_{5}=\int_{0}^{1}\left[Y(\xi) \varphi^{\prime}(\xi)-\varphi(\xi) Y^{\prime}(\xi)+\varphi^{2}(\xi)\right] d \xi .
\end{aligned}
$$

Taking into account Eqs. (7)-(8), and considering the boundary and the compatibility conditions, $H_{1}=0$ and $H_{2}, H_{3}, H_{4}$, we get

$$
\begin{aligned}
& H_{2}=\mu^{2} s^{2} Y(0) \varphi(0) \\
& H_{3}=-\frac{1}{\varepsilon}\left[s^{2} \eta_{m}\left[\varphi^{\prime}(\beta)\right]^{2}+\frac{\eta_{q} r^{2}}{s^{2}}\left[Y^{\prime}(\beta)-\left(1+F_{c}^{2} s^{2}\right) \varphi(\beta)\right]^{2}\right] \\
& H_{4}=-\mu^{2} s^{2} Y(1) \varphi(1) .
\end{aligned}
$$

Therefore, from Eqs. (54), (72), (77) and (78), a closed-form expression for $F_{c}^{*}$ can be reached as

$$
F_{c}^{* 2}=F_{c}^{2}+\frac{\eta_{m}\left[\varphi^{\prime}(\beta)\right]^{2}+\eta_{q}\left(r^{2} / s^{4}\right)\left[Y^{\prime}(\beta)-\left(1+F_{c}^{2} s^{2}\right) \varphi(\beta)\right]^{2}}{Y(0) \varphi(0)-Y(1) \varphi(1)+\int_{0}^{1}\left[Y(\xi) \varphi^{\prime}(\xi)-\varphi(\xi) Y^{\prime}(\xi)+\varphi^{2}(\xi)\right] d \xi} .
$$

From the mode shapes corresponding to the boundary conditions, Eqs. (55)-(59), it can be seen that the denominator is different from zero. Note that the difference of buckling loads are proportional to the square of the bending moment and shear force transmitted by the cracked section.

This method provides a closed expression for the first buckling load of Timoshenko cracked columns with simple boundary conditions from the well-known buckling modes $Y(\xi), \varphi(\xi)$ of the uncracked column.

\subsection{Shear component proportional to the total slope}

From Eqs. (39)-(42), the first-order perturbative terms take the form:

$$
\begin{aligned}
& \left(1-F_{c}^{2} s^{2}\right) V_{1}^{\prime \prime}(\xi)-\psi_{1}^{\prime}(\xi)=\mu^{2} s^{2} y^{\prime \prime}(\xi) \quad 0<\xi<\beta \\
& s^{2} \psi_{1}^{\prime \prime}(\xi)+V_{1}^{\prime}(\xi)-\psi_{1}(\xi)=0 \quad 0<\xi<\beta \\
& \left(1-F_{c}^{2} s^{2}\right) V_{2}^{\prime \prime}(\xi)-\psi_{2}^{\prime}(\xi)=\mu^{2} s^{2} y^{\prime \prime}(\xi) \quad \beta<\xi<1 \\
& s^{2} \psi_{2}^{\prime \prime}(\xi)+V_{2}^{\prime}(\xi)-\psi_{2}(\xi)=0 \quad \beta<\xi<1
\end{aligned}
$$

with boundary conditions at the end supports $\left(\xi_{s}=0, \xi_{s}=1\right)$ :

- Fixed end: $V_{i}\left(\xi_{s}\right)=0, \psi_{i}\left(\xi_{s}\right)=0$

- Simply supported end: $V_{i}\left(\xi_{s}\right)=0, \psi_{i}^{\prime}\left(\xi_{s}\right)=0$

- Free end: $\psi_{i}^{\prime}\left(\xi_{s}\right)=0,\left(1-F_{c}^{2} s^{2}\right) V_{i}^{\prime}\left(\xi_{s}\right)-\psi_{i}\left(\xi_{s}\right)=\mu^{2} s^{2} y^{\prime}\left(\xi_{s}\right)$

and compatibility conditions at the cracked section $(\xi=\beta)$ :

- $V_{2}(\beta)-V_{1}(\beta)=\left[\left(\eta_{q} r^{2}\right) /\left(\varepsilon s^{2}\right)\right] \cdot\left[\left(1-F_{c}^{2} s^{2}\right) Y^{\prime}(\beta)-\varphi(\beta)\right]$

- $\psi_{2}(\beta)-\psi_{1}(\beta)=\eta_{m} \varphi^{\prime}(\beta) / \varepsilon$

- $\psi_{1}^{\prime}(\beta)=\psi_{2}^{\prime}(\beta)$

- $\left(1-F_{c}^{2} s^{2}\right) V_{1}^{\prime}(\beta)-\psi_{1}(\beta)=\left(1-F_{c}^{2} s^{2}\right) V_{2}^{\prime}(\beta)-\psi_{2}(\beta)$

and when the same method explained above is followed, the closed-form expression for $F_{c} *$ now takes the form:

$$
F_{c}^{* 2}=F_{c}^{2}+\frac{\eta_{m}\left[\varphi^{\prime}(\beta)\right]^{2}+\eta_{q}\left(r^{2} / s^{4}\right)\left[\left(1-F_{c}^{2} s^{2}\right) Y^{\prime}(\beta)-\varphi(\beta)\right]^{2}}{Y(0) Y^{\prime}(0)-Y(1) Y^{\prime}(1)+\int_{0}^{1} Y(\xi) Y^{\prime \prime}(\xi) d \xi}
$$

proportional, also in this case, to the square of the bending moments and shear forces transmitted by the cracked section. As in the previous case, note that the denominator of the above expression never vanish.

\section{Results}

\subsection{Application to simply supported cracked columns}

\subsubsection{Shear component proportional to the bending rotation}

For the particular case of a simply supported Timoshenko cracked column with different crack severities, $\eta_{m}$, and cracked section positions, $\beta$, the aforementioned methods have been applied. 
The direct method (Section 3 ) was implemented by substituting the boundary conditions for the simply supported column, (Eq. (17)) and the compatibility conditions, (Eqs. (67)-(70)), in Eqs. (43)-(46), and the critical buckling load $F_{c}^{*}$ that satisfies the condition Eq. (48) was calculated.

Alternatively, the critical buckling load can be obtained using the proposed first-order perturbative solution. The mode shapes for a uniform simply supported uncracked Timoshenko column take the form given in Eq. (55). The corresponding first critical buckling load (for an uncracked simply supported column), $F_{c}$, can be calculated from Eq. (13) with $\Omega=\pi$. When these modes are substituted in Eq. (79), the following expression for the first critical load for a weakened column, $F_{c}^{* 2}$, is obtained:

$$
F_{c}^{* 2}=F_{c}^{2}-2 \eta_{m} \frac{[\pi \sin (\pi \beta)]^{2}}{1+2 F_{c}^{2} s^{2}} .
$$

In this case, the shear force is null in the whole column and only bending moment is transmitted by the cracked section affecting the calculated critical buckling load.

\subsubsection{Shear component proportional to the total slope}

When the expressions of transverse deflection and slope given by Eq. (55) are introduced into Eq. (84), the closed form expression for the first critical buckling load for simply supported cracked columns and shear-force, taking into account the second approach, takes the form

$$
F_{c}^{* 2}=F_{c}^{2}-2 \eta_{m}\left[\pi\left(1-F_{c}^{2} s^{2}\right) \sin (\pi \beta)\right]^{2}
$$

where $F_{c}$ is calculated from Eq. (38) with $\Omega=\pi$.

Again, the shear force is equal to zero at any section of the column and only the transmitted bending moment affects the calculated critical buckling load.

However, note that the first critical load is slightly different from that calculated by the previous model. The reason is that both critical load for the uncracked column, bending moment transmitted by the cracked section, as well as the denominator of Eq. (84), differ from those corresponding to that obtained with the hypothesis that the shear force is proportional to the bending rotation.

\subsubsection{Comparison between both shear component definitions}

For a column with $W / L=0.15, r^{2}=0.0019$ and $s^{2}=0.00585$, the first buckling load $F_{c}^{*}$, has been calculated by means of both shear consideration described above for different cracked section locations, $\beta$, and for different values of $\eta_{m}$ (depending on this parameter to the width-to-length ratio $W / L$ and crack-to-width ratio $\alpha$, Eqs. (21), (23)). For example, in a column with the indicated properties, where $\alpha$ is in the range [0-0.50], the corresponding crack severity parameter $\eta_{m}$ is in the range [0-0.513].

Fig. 2(a) and 2(b) show the variation of the critical buckling load with the crack severity $\left(\eta_{m}\right)$ for cracks located at a quarter of the mid-span $(\beta=0.25)$ and at middle section $(\beta=0.50)$, respectively. The buckling load was normalized, corresponding to an uncracked simply supported Euler-Bernoulli column, $F_{0}=\pi$. From these figures, it can be seen that the first-order solutions, considering shear component proportional to the bending rotation, Eq. (85), and proportional to the total slope, Eq. (86), are practically identical.

The difference between the first-order buckling loads and those obtained by means of the direct method, considering the Timoshenko Beam Theory with the shear component proportional to the bending rotation (TBT Direct solution) is less than 5\% when $\eta_{m} \leq 0.27$ (corresponding to $\alpha \simeq 0.40$ ) for a crack located at $\beta=0.25$, when $\eta_{m} \leq 0.13(\alpha \simeq 0.30)$ for a crack located at $\beta=0.50$.

It is worth noting that defining the shear force proportional to the total slope, the buckling loads obtained virtually coincides with those calculated with the first definition of the shear component, and therefore are not shown in the figures. The shear force effect can be appreciated comparing with the results obtained by considering the Euler-Bernoulli Beam Theory (EBBT Direct solution [11]).

\subsection{Application to cantilever cracked columns}

\subsubsection{Shear component proportional to the bending rotation}

According to the perturbative method proposed, and using the mode shapes of transverse deflection and slope given by Eq. (57) into Eq. (79), the expression for the first critical load in the case of a cantilever cracked column is

$$
F_{c}^{* 2}=F_{c}^{2}-\frac{\eta_{m}}{2} \frac{\left[\pi \cos \left(\frac{\pi \beta}{2}\right)\right]^{2}}{1+2 F_{c}^{2} s^{2}}
$$

with $F_{c}$ calculated from Eq. (13) with $\Omega=\pi / 2$.

As in the pinned-pinned column, only the bending moment transmitted by the cracked section is considered to evaluate the critical load of the weakened column, due to the nullity of the shear force at any section. 

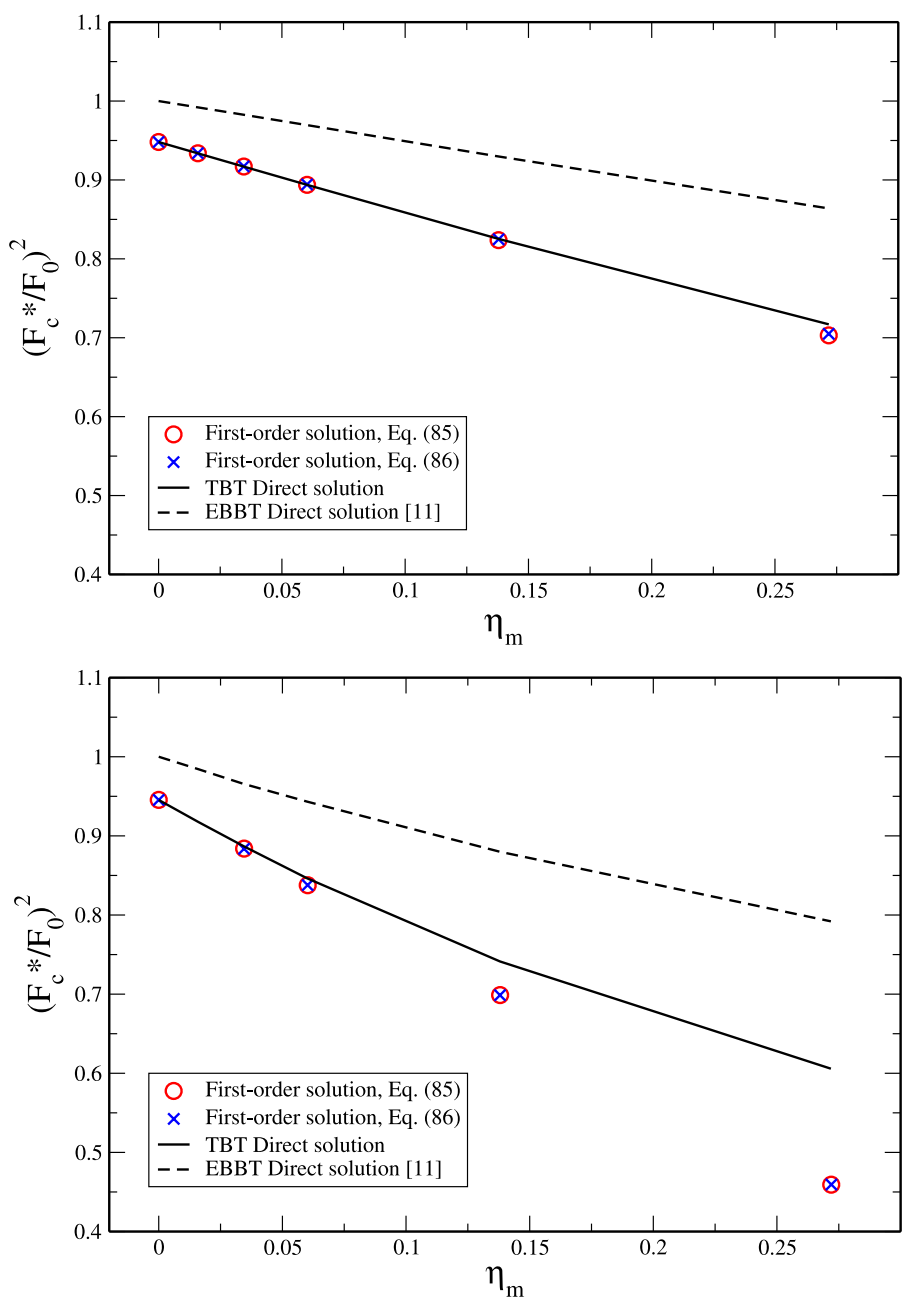

Fig. 2. First buckling load for different values of crack severity for a cracked simply supported column. Crack located at (a) $\beta=0.25$ and (b) $\beta=0.50$.

\subsubsection{Shear component proportional to the total slope}

In the case of the shear component proportional to the total slope, the first critical buckling load for a cantilever cracked column using the perturbation method has the form:

$$
F_{c}^{* 2}=F_{c}^{2}-\frac{\eta_{m}}{2}\left[\pi^{2}\left(1-F_{c}^{2} s^{2}\right)^{2} \cos ^{2}\left(\frac{\pi \beta}{2}\right)\right]
$$

where $F_{c}$ is obtained from Eq. (38) with $\Omega=\pi / 2$.

Again, the shear force is zero and only the bending moment is considered to evaluate the critical buckling load.

\subsubsection{Comparison between both shear component definitions}

For the same column characteristics and crack locations as in the previous study, the variation of $F_{c}^{*}$ obtained from the first-order solution for both shear component definitions, Eqs. (87) and (88), (normalized with the buckling load corresponding to an uncracked cantilever Euler-Bernoulli column, $F_{0}=\pi / 2$ ) with $\eta_{m}$ is shown in Fig. 3(a) and (b). The first-order buckling loads, considering both shear component definitions, are very close to each other. Differences with respect to the results obtained from the TBT direct solution (in this case also, both shear models lead to practically the same buckling loads) are less than $5 \%$ when $\eta_{m} \leq 0.13$, for crack located at $\beta=0.25$, and $\eta_{m} \leq 0.27$ for a crack located at $\beta=0.50$.

The influence of the shear component in a cantilever column can be seen comparing with the results obtained considering the Euler-Bernoulli Beam Theory (EBBT Direct solution [11]). 

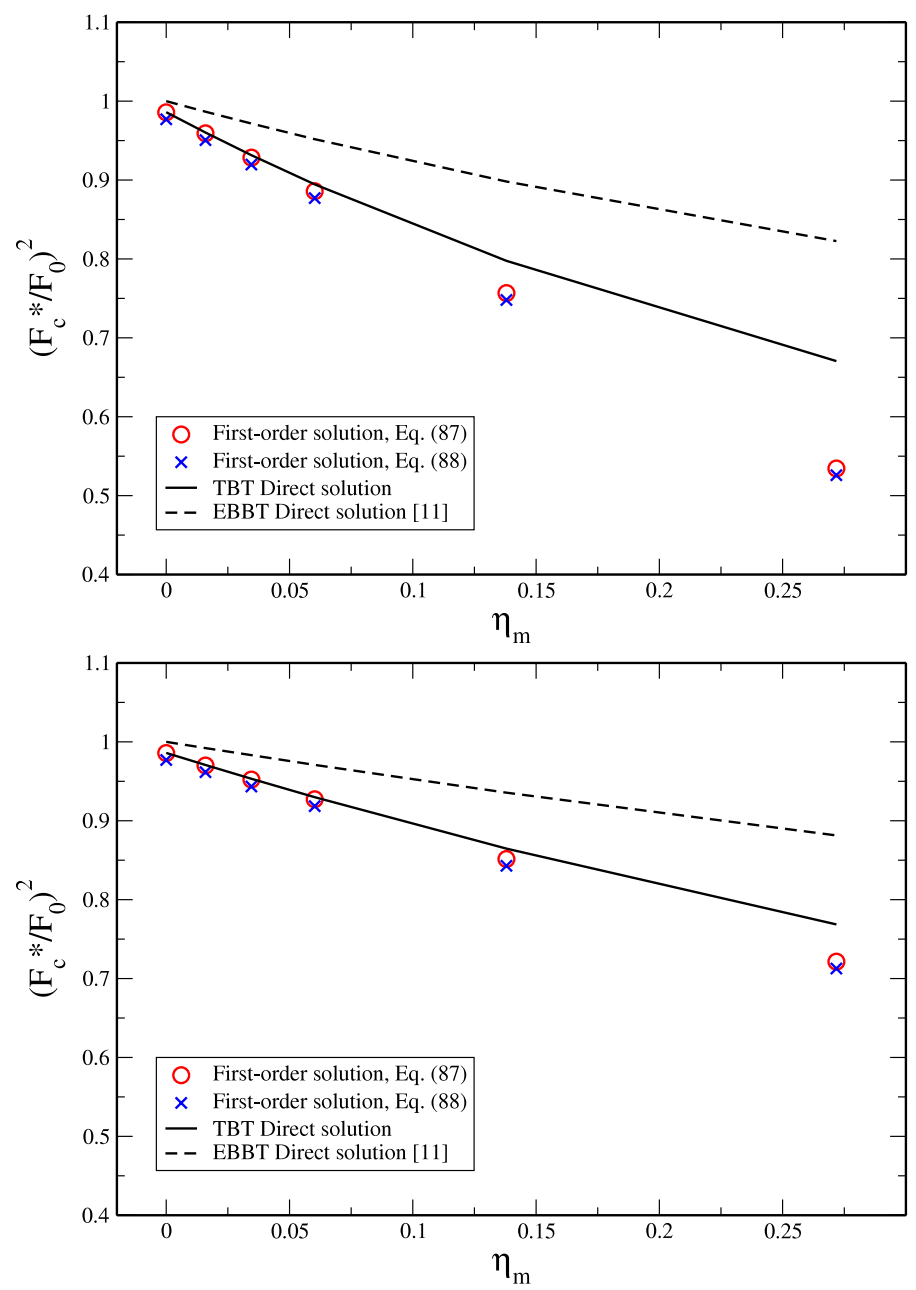

Fig. 3. First buckling load for different values of crack severity for a cracked cantilever column. Crack located at (a) $\beta=0.25$ and (b) $\beta=0.50$.

\subsection{Application to clamped-pinned cracked columns}

In the cases presented above, shear force is null in the whole column due to the boundary conditions considered. However, in the case of a clamped-pinned column both bending moment and shear force are present at the cracked section and the influence of both effects can be considered together.

\subsubsection{Shear component proportional to the bending rotation}

If the shear component is considered proportional to the bending rotation, the first critical buckling load, Eq. (79), for a clamped-pinned cracked column can be written as

$$
F_{c}^{* 2}=F_{c}^{2}\left[1-2 F_{c}^{2} \cdot \frac{\eta_{m} \cdot \Omega_{c} \sin ^{2}\left[(1-\beta) \Omega_{c}\right]+\eta_{q} \cdot r^{2} \Omega_{c} \sin ^{2}\left(\Omega_{c}\right)}{\tan \left(\Omega_{c}\right)\left[2 \Omega_{c}^{2}-\Omega_{c} \tan \left(\Omega_{c}\right)-\sin ^{2}\left(\Omega_{c}\right)\right]}\right]
$$

where $F_{c}$ and $\Omega_{c}$ are calculated solving the following system of equations: $\Omega_{c}=F_{c} \cdot\left(1+F_{c}^{2} s^{2}\right)^{0.5}$ and $\tan \left(\Omega_{c}\right)=\Omega_{c} /$ $\left(1+F_{c}^{2} s^{2}\right)$.

\subsubsection{Shear component proportional to the total slope}

If the shear component is considered proportional to the total slope, (Eq. (84)) the first critical buckling load for a clamped-pinned cracked column takes the form

$$
F_{c}^{* 2}=F_{c}^{2}\left[1-2 F_{c}^{2} \cdot \frac{\eta_{m} \cdot \sin ^{2}\left[(1-\beta) \Omega_{c}\right]+\eta_{q} \cdot r^{2} \sin ^{2}\left(\Omega_{c}\right)}{\Omega_{c}^{2}+\cos \left(2 \Omega_{c}\right)+\Omega_{c} \cos \left(\Omega_{c}\right) \sin \left(\Omega_{c}\right)-1}\right]
$$

where $F_{c}$ and $\Omega_{c}$ were calculated from $\Omega_{c}=F_{c} \cdot\left(1-F_{c}^{2} s^{2}\right)^{-0.5}$, and $\tan \left(\Omega_{c}\right)=\Omega_{c} \cdot\left(1-F_{c}^{2} s^{2}\right)$. 

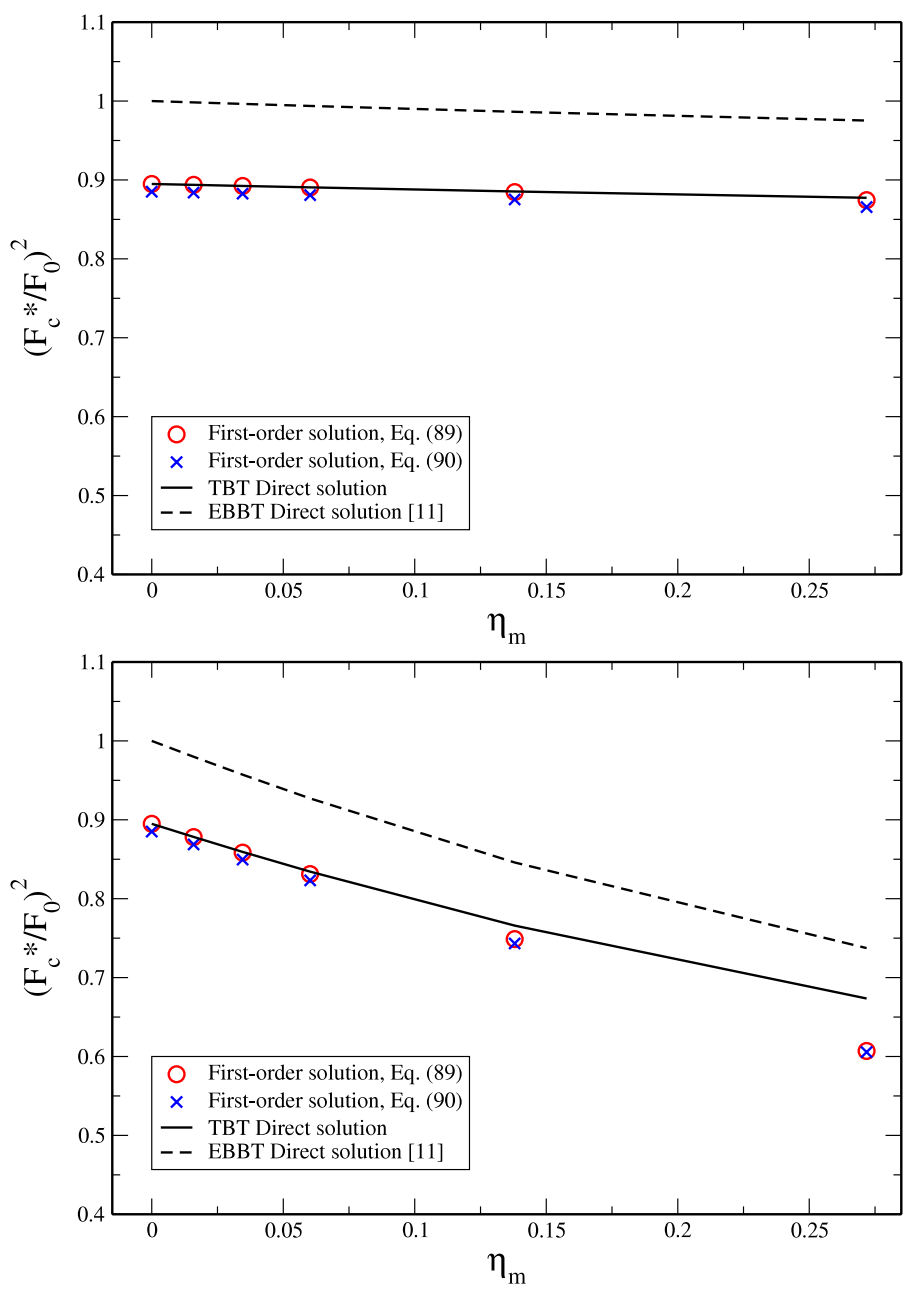

Fig. 4. First buckling load for different values of crack severity for a cracked clamped-pinned column. Crack located at (a) $\beta=0.25$ and (b) $\beta=0.50$.

\subsubsection{Comparison between both shear component definitions}

For a clamped-pinned cracked column with same characteristics and crack locations as in previous cases, $F_{c}^{*}$ (normalized with the first buckling load corresponding to an uncracked clamped-pinned Euler-Bernoulli column, $F_{0}=h \pi$ with $h=1 / \sqrt{0.699}$ ) were obtained for different crack severities and crack location from the perturbative method solutions for both shear component definitions, (89) and (90).

Due to the direct relation between $\eta_{m}$ and $\eta_{q}$ through the geometric relation $W / L$ and crack-to-width ratio $\alpha$ (see Eqs. (21)-(23)), the $F_{c}^{*}$ results for both crack locations are presented in Fig. 4(a) and (b) as a function of $\eta_{m}$. Both shear models lead to practically the same buckling loads. Differences with respect to the results obtained from the TBT direct solution is less than $5 \%$ when $\eta_{m} \leq 0.97$ (correspond to $\alpha=0.60$ ) for a crack located at $\beta=0.25$, and $\eta_{m} \leq 0.27$ (correspond to $\alpha=0.40$ ) for a crack located at $\beta=0.50$.

The influence of the shear component in a clamped-pinned column can be seen comparing with the results obtained considering the Euler-Bernoulli Beam Theory (EBBT Direct solution [11]).

\section{Conclusions}

This work provides direct and perturbative solutions for determining the buckling loads of weakened Timoshenko columns with different boundary conditions, and different considerations of the shear component: proportional to the bending rotation or to the total slope.

The crack model used considers the weakened column as two segments connected by two massless springs (one extensional and another rotational). The differential equations for the buckling are established and solved individually for each segment with the corresponding boundary conditions and the appropriate compatibility conditions at the cracked section, including discontinuities in the rotation due to bending moment as well as in the transverse deflection due to shear force. 
Cracked columns with several boundary conditions, different cracked section locations $\beta$ and different crack severities $\eta_{m}$ have been considered. The first buckling load has been obtained by the first-order perturbation method and compared with those reached by the direct solution.

For the cases of simply supported and cantilever cracked columns, the results obtained show a good agreement between both methods for shallow cracks, with a difference less than $5 \%$ for $\eta_{m} \leq 0.13$ (corresponding to $\alpha \leq 0.3$ with $W / L=0.15$ ). For these cases, the shear force is null in the whole column and the cracked section transmits only bending moment. However, different definitions of the shear component have a direct influence on the corresponding $F_{c}^{*}$ expression, as well as on the first buckling load of the uncracked column $F_{c}$, giving slight differences in the solution.

In the clamped-pinned case, where the critical load of the weakened column gathers both bending moment and shear force terms, the differences between the first critical buckling load obtained with the direct method and the perturbation techniques are less than $5 \%$ for $\eta_{m} \leq 0.27$ for the crack locations analysed.

Attending to the results obtained by the direct and perturbative methods presented, differences due to the shear component definitions are minimal.

The perturbation method provides closed-form expressions for the critical buckling load of Timoshenko cracked columns with different boundary conditions and different definitions of the shear component.

\section{Acknowledgements}

The authors would like to thank the Comisión Interministerial de Ciencia y Tecnología of the Spanish Government and to the Comunidad Autónoma de Madrid for partial support of this work through the research projects DPI2011-23191 and CCG10-UC3M-DPI-5596, respectively.

\section{References}

[1] S.P. Timoshenko, J.M. Gere, Theory of Elastic Stability, McGraw-Hill, New York, 1961

[2] J. Aristizábal-Ochoa, Column stability and minimum lateral bracing: effects of shear deformations, Journal of Engineering Mechanics-ASCE 130 (10) (2004) 1223-1232.

[3] J. Aristizábal-Ochoa, Timoshenko beam-column with generalized end conditions and nonclassical modes of vibration of shear beams, Journal of Engineering Mechanics-ASCE 130 (10)(2004) 1151-1159.

[4] L. Freund, G. Herrmann, Dynamic fracture of a beam or plate in bending, Journal of Applied Mechanics 98 (1976) 112-116.

[5] H. Okamura, K. Watanabe, T. Takano, Applications of compliance concept in fracture mechanics, in: Ch. J.G. Kaufman (Ed.), Progress in Flaw Growth and Fracture Toughness Testing, Vol. 536, American Society for Testing and Materials, Philadelphia, 1973, pp. 423-438.

[6] T. Tharp, A finite element for edge-cracked beam columns, International Journal for Numerical Methods in Engineering 24 (1987) 1941-1950.

[7] C.Y. Wang, C.M. Wang, T. Aung, Buckling of a weakened column, Journal of Engineering Mechanics-ASCE 130 (2004) $1373-1376$.

[8] S. Caddemi, I. Calió, Exact solution of the multi-cracked Euler-Bernoulli column, International Journal of Solids and Structures 45 (2008) $1332-1351$.

[9] B. Biondi, S. Caddemi, Closed form solutions of Euler-Bernoulli beams with singularities, International Journal of Solids and Structures 42 (2005) 3027-3044.

[10] B. Biondi, S. Caddemi, Euler-Bernoulli beams with multiple singularities in the flexural stiffness, European Journal of Mechanics A/Solids 26 (2007) 789-809.

[11] J.A. Loya, G. Vadillo, J. Fernández-Sáez J, First-order solutions for the buckling loads of Euler-Bernoulli weakened colums, Journal of Engineering Mechanics-ASCE 136 (5) (2010) 674-679.

[12] L. Arboleda-Monsalve, D. Zapata-Medina, J. Aristizabal-Ochoa, Stability and natural frequencies of a weakened Timoshenko beam-column with generalized end conditions under constant axial load, Journal of Sound and Vibration 307 (2007) 89-112.

[13] D. Zapata-Medina, L. Arboleda-Monsalve, J. Aristizábal-Ochoa, Static stability formulas of a weakened Timoshenko column: effects of shear deformations, Journal of Engineering Mechanics-ASCE 136 (2010) 1528

[14] Q. Li, Buckling of multi-step cracked columns with shear deformation, Engineering Structures 23 (2001) 356-364.

[15] I. Takahashi, Vibration and stability of non-uniform cracked Timoskenko beam subjected to follower force, Computer and Structures 71 (1999) 585-591.

[16] Q. Li, Classes of exact solutions for buckling of multi-step non-uniform columns with an arbitrary number of cracks subjected to concentrated and distributed axial loads, International Journal of Engineering Science 41 (2003) 569-586.

[17] J.A. Loya, L. Rubio, J. Fernández-Sáez, Natural frequencies for bending vibrations of Timoshenko cracked beams, Journal of Sound and Vibration 290 (2006) 640-663.

[18] R. Adams, P. Cawley, C. Pye, B. Stone, A vibration technique for nondestructive assesing the integrity of structures, Journal of Mechanical Engineering Science 20 (1978) 93-100.

[19] A. Morassi, Crack-induced changes in eigenfrecuencies of beam structures, Journal of Engineering Mechanics-ASCE 119 (1993) 1768-1803

[20] Y. Narkis, Identification of crack location in vibrating simply supported beams, Journal of Sound and Vibration 172 (1994) 549-558.

[21] J. Fernández-Sáez, L. Rubio, C. Navarro, Approximate calculation of the fundamental frequency for bending vibrations of cracked beams, Journal of Sound and Vibration 225 (1999) 345-352.

[22] J. Fernández-Sáez, C. Navarro, Fundamental frequency of cracked beams: an analytical approach, Journal of Sound and Vibration 256 (2002) 17-31.

[23] M. Krawczuk, M. Palacz, W. Ostachowicz, The dynamic analysis of cracked Timoshenko beams by spectral element method, Journal of Sound and Vibration 264 (2003) 1139-1153.

[24] A. Morassi, Identification of a crack in a rod based on changes in a pair of natural frequencies, Journal of Sound and Vibration 242 (4) (2001) 577-596.

[25] M. Dilena, A. Morassi, The use of antiresonances for crack detection in beams, Journal of Engineering Mechanics-ASCE 276 (1-2) (2004) 195-214.

[26] H. Tada, P. Paris, G. Irwin, The Stress Analysis of Cracks Handbook, second ed., Paris Productions, Inc., St Louis, 1985.

[27] B. Geist, J. McLaughlin, Double eigenvalues for the uniform Timoshenko beam, Applied Mathematics Letters 10 (1997) $129-134$.

[28] N. van Rensburg, A. van der Merwe, Natural frequencies and modes of a Timoshenko beam, Wave Motion 44 (2006) 58-69.

[29] R. Courant, D. Hilbert, Methods of Mathematical Physics, Vol. 1, McGraw-Hill, New York, 1953. 